\title{
A 2.8-7.5 pJ/Pulse Highly-Flexible Impulse-Radio Ultra-Wideband Pulse-Generator
}

\author{
Kin Keung Lee* and Tor Sverre Lande
}

\begin{abstract}
A low-power on-off-keying impulse-radio (IR) ultra-wideband (UWB) pulse generator (PG) intended for wireless-powered IR-UWB radio applications is presented. The proposed PG has high flexibility, the center frequency, output power and pulse-width (PW) are controllable depending on channel conditions and data rates. Qualitative frequency-domain and transient analyses are presented. A new figure-of-merit (FoM) is proposed such that a more precise comparison between different PGs can be made. The PG is successfully implemented in a TSMC $90 \mathrm{~nm}$ CMOS process, measurements show the energy consumption and FoM to be $2.8-7.5 \mathrm{pJ} /$ pulse and $1.6-2.6 \%$ respectively. The output swing and PW are 277-329 $\mathrm{mV}_{\mathrm{p}-\mathrm{p}}$ and 509-1088 ps respectively. The core area is $0.092 \mathrm{~mm}^{2}$.
\end{abstract}

\section{INTRODUCTION}

Since the Federal Communication Commission (FCC) released a large spectral mask (i.e., 3.1-10.6 GHz) for unlicensed uses, ultra-wideband (UWB) technology has been an active research field. The wide spectral mask not only enables higher date rate, but also precise localization ability and constructing energy-efficient transmitters (TX) by using impulse-radio (IR) technology. Implementations of highperformance wireless-powered IR-UWB TXs become possible even in standard CMOS processes. Pulse generator $(\mathrm{PG})$ is usually the most energy-demanding component in such wireless systems. Large energy dissipation will reduce the TX sensitivity and increase the difficulty of power management circuits design. In most wireless-powered IR-UWB TXs [1-3], the PG energy dissipation is less than $10 \mathrm{pJ} / \mathrm{pulse}$. In addition, the PG efficiency is also important to provide reasonable emitted energy and, hence, good communication distance.

The basic idea of most published IR-UWB PGs is to use a high-frequency waveform generator (HFWG), which can be an oscillator, phase-locked loop or delay-line (DL) etc., and shape the output signal to generate the desired envelope by mainly three approaches: filtering [4], spectrum mixing $[5,6]$ or multi-pulse combination (MPC) $[7,8]$. For the filtering approach, the HFWG output signal is coupled to and filtered by a pulse shaping filter so that the resultant signal can meet the spectrum regulations. In additional to the circuit simplicity, another advantage is that the output parasitic capacitance, which is usually the dominating one inside the PG, can be used as part of the filter and tuned-out by inductors inside the filter. In other words, the capacitive and switching losses could be reduced. However, the filtered energy may be wasted and on-chip spiral inductors are area-demanding.

The spectrum mixing approach consists of a local oscillator (LO) and a mixer like conventional narrowband TXs, the high-frequency LO output is then mixed with the envelope shape. The power consumption and area are relatively large because more components are needed compared to the other two approaches. Moreover, the leakage from LO to the antenna could be problematic. However, a definite advantage is the ability to generate high-quality bi-phase shift key (BPSK) IR-UWB signals. The mixing function could also be performed by switching the oscillator on and off (will be referred to as switching oscillator, SO, approach) [9]. This eliminates the mixer and LO leakage, but reduces control

\footnotetext{
Received 3 November 2014, Accepted 13 December 2014, Scheduled 21 December 2014

* Corresponding author: Kin Keung Lee (kklee@ifi.uio.no).

The authors are with the Department of Informatics, University of Oslo, Norway.
} 
of emitted pulse shape. The SO approach is reported to be energy efficient because of its simplicity. Nevertheless the low antenna resistance loads the tank and reduces its quality factor (QF), large bias current is needed to start-up the oscillation and obtain a large output swing [9]. This makes the SO approach less suitable for wireless-powered IR-UWB radios.

MPC IR-UWB PGs usually utilize DLs as the HFWG. Based on the propagation delay (PD) of the DL and output drivers with different strength, Gaussian-approximate waveforms can be obtained. The MPC approach is shown to be very energy-efficient (5.2 pJ/pulse has been reported in [7]) and small-size because it contains mainly digital gates. Nevertheless most of the MPC IR-UWB PGs operate at the low UWB band, this is because the capacitive and switching losses due to the output parasitic capacitance increase with frequency. Also, it is not easy to control the envelope accurately due to process variations, filtering and/or calibration may be required.

A low-power DL-based MPC on-off-keying (OOK) IR-UWB PG is presented in this paper. It uses an on-chip spiral inductor to tune out the output parasitic capacitance, the driving requirement and, hence, the transistor size of the output drivers are reduced. In other words, the capacitive and switching losses are also reduced which makes the proposed PG energy-efficient. Moreover, the center frequency $\left(f_{C}\right)$, output power and pulse-width $(\mathrm{PW})$ are adjustable depending on channel conditions and data rates. A similar structure has been adopted in [1], however limited analysis and results have been presented. The proposed PG is realized in a TSMC $90 \mathrm{~nm}$ CMOS process and shows good measurement results.

\section{IR-UWB SIGNAL GENERATION}

The basic idea of the proposed PG is to inject energy into an RLC tank which consists of the antenna resistance $\left(R_{A N T}\right)$ periodically as shown in Figure 1(a). The tank resonant frequency $\left(f_{o}\right)$ and quality factor $\left(Q_{T}\right)$ are given as:

$$
\begin{aligned}
f_{o} & =\frac{1}{2 \pi \sqrt{L_{T} C_{T}}} \\
Q_{T} & =\frac{R_{A N T}}{\omega_{o} L_{T}}=\omega_{o} C_{T} R_{A N T}
\end{aligned}
$$

Assume that $N$ energy pulses with $50 \%$ duty-cycle are injected to the tank by turning on and off the switch periodically with a frequency of $f_{S W}$ and that every single injected energy pulse circulates back and forth inside the tank with $f_{o}$. Due to the $R_{A N T}$, the $i$-th resonating pulse decays as:

$$
V_{R, i \text {-th }}=V_{P} \exp \left(-\frac{f_{o}}{Q_{T}} t\right) \sin \left(2 \pi f_{o} t\right)
$$

The signal dies out in approximately $Q_{T}$ cycle. If $f_{S W}$ and $f_{o}$ are the same, the resonating pulses will be in-phase and added together. As a result, a higher-energy and longer-PW IR-UWB signal can be obtained. An example with $Q_{T}=2$ and $N=2$ is shown in Figure 1(b).

It is difficult to determine the output signal amplitude because of the system nonlinearities. Nevertheless it is important to estimate the system frequency response so that the FCC part 15 mask requirements can be met. A qualitative frequency domain analysis is performed. The waveform of the

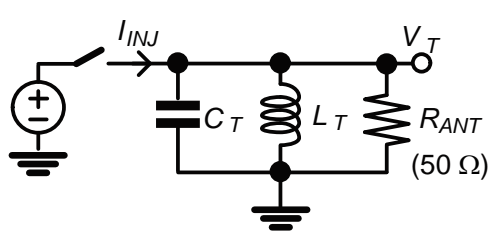

(a)

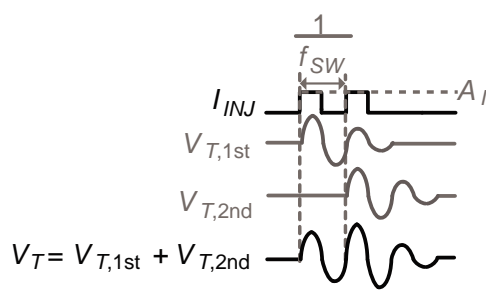

(b)

Figure 1. (a) Proposed system of the PG and (b) its timing waveforms. 


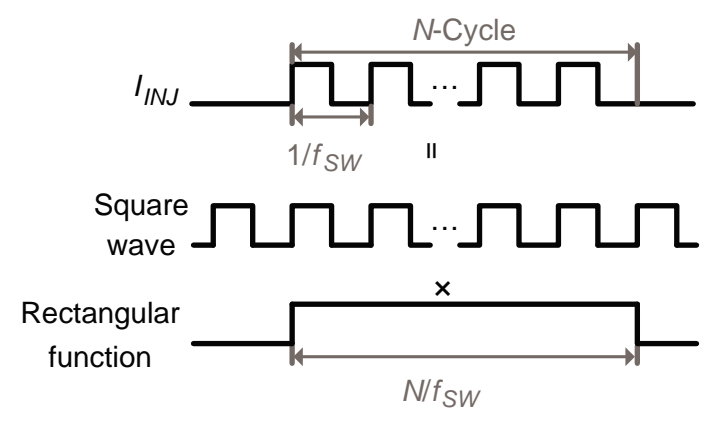

Figure 2. Timing waveforms related to $I_{I N J}$.

injected current $\left(I_{I N J}\right)$ can be modeled as a square wave with a frequency of $f_{S W}$ multiplying with a rectangular function with a width of $\frac{N}{f_{S W}}$ as shown in Figure 2. As a result, the resultant frequency spectrum can be expressed as

$$
I_{I N J}(f)=\overbrace{\frac{4 A_{I}}{\pi} \sum_{k=1}^{\infty} \frac{\delta\left(2 \pi(2 k-1) f_{S W}\right)}{2 k-1}}^{\text {Square wave }} * \overbrace{\frac{N}{f_{S W}} \operatorname{sinc}\left(\frac{N f}{f_{S W}}\right)}^{\text {Rectangular function }}
$$

where $A_{I}$ is the amplitude of the injected current. Because the higher-order components of the square wave (the first term) are always out of the UWB band (for example, if $f_{S W}=4 \mathrm{GHz}$, the higher-order components will be $\geq 12 \mathrm{GHz}$ ), only the fundamental component is considered and $I_{I N J}$ becomes

$$
I_{I N J}(f)=\frac{4 N \cdot A_{I}}{\pi f_{S W}}\left[\delta\left(2 \pi f_{S W}\right) * \operatorname{sinc}\left(\frac{N f}{f_{S W}}\right)\right]
$$

Its bandwidth $(\mathrm{BW})$ is determined by the sinc function and the $-10-\mathrm{dB} \mathrm{BW}\left(B W_{-10 \mathrm{~dB}}\right)$ of $I_{I N J}^{2}$ is found to be $\frac{f_{S W}}{N}$ approximately.

$I_{I N J}$ is then filtered by the RLC tank. One important parameter is $Q_{T}$, and large $Q_{T}$ provides narrower BW and reduces the sideband energy of $I_{I N J}$. Nevertheless the in-band energy may also be filtered-out and the energy efficiency is reduced. Also $Q_{T}$ has to be much smaller than the inductor QF $\left(Q_{L}\right)$ so as to prevent insertion losses which is given as [10]

$$
\mathrm{IL}=\frac{1}{1+\frac{Q_{T}}{Q_{L}}}
$$

The QF of on-chip spiral inductors is normally around 10-20. Because the energy efficiency is of the most important in this work and the low-frequency (LF) sideband can be filtered-out by adding highpass filter (HPF) or/and exploring the antenna bandpass property, small $Q_{T}$ is preferred to minimize the insertion loss. Notice that the data rate and localization ability also improve with increased BW $\left(\right.$ small $Q_{T}$ ) [1]. A rule of thumb is to set $B W_{-10 \mathrm{~dB}}$ of $I_{I N J}^{2}$ to be smaller than or equal to the $-3-\mathrm{dB}$ BW of the RLC tank such that the tank provides only insignificant attenuation. In other words,

$$
\frac{f_{S W}}{N} \leq \frac{f_{o}}{Q_{T}} \Rightarrow N \geq Q_{T}
$$

An example is shown in Figure 3, which shows system-level simulation results with $f_{o}=f_{S W}=$ $5 \mathrm{GHz}$ and different combinations of $Q_{T}$ and $N$. The peak power spectral densities (PSD) are normalized to $-41.3 \mathrm{dBm} / \mathrm{MHz}$ for better comparisons. In Figure 3(a), $N$ is set to 2 . Increasing $Q_{T}$ from 2 to 5 can reduce the $\mathrm{LF}$ sideband energy for $7.5 \mathrm{~dB}$, but reduce $B W_{-10 \mathrm{~dB}}$ for $32 \%$ (from $3.1 \mathrm{GHz}$ to $2.1 \mathrm{GHz}$ ). If $N$ is increased to 5 as shown in Figure 3(b), increasing $Q_{T}$ from 2 to 5 only reduces $B W_{-10 \mathrm{~dB}}$ for $14 \%$ (from $1.4 \mathrm{GHz}$ to $1.2 \mathrm{GHz}$ ). Notice that increasing $N$ can provide higher attenuation to the sideband energy because of the narrower BW of the sinc function, the trade-off is that the BW of the mainlobe is also reduced. 


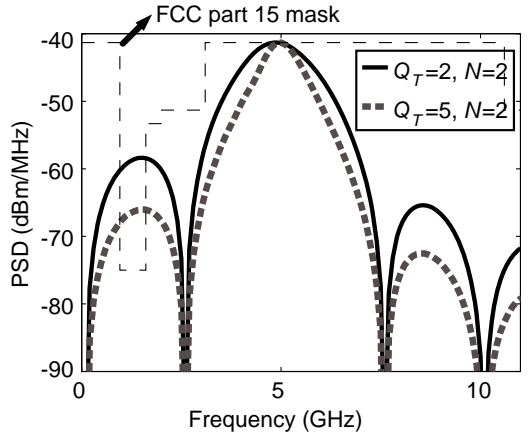

(a)

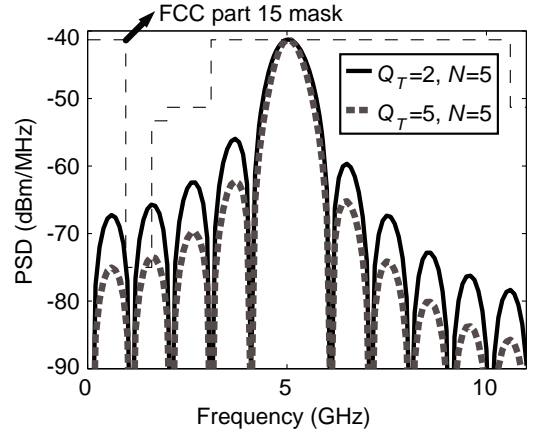

(b)

Figure 3. System-level simulated PSD with different $Q_{T}$. (a) $N=2$ and (b) $N=5$.

\section{PROPOSED IR-UWB PG}

The schematic of the proposed IR-UWB PG is shown in Figure 4(a). LF_EN = ' 0 ' and PW_EXT $=$ ' 1 ' are assumed at this moment, the setting will be explained later. Instead of using a single switch driven by an oscillator as shown in Figure 1(a), several switches are cascaded and turned on and off

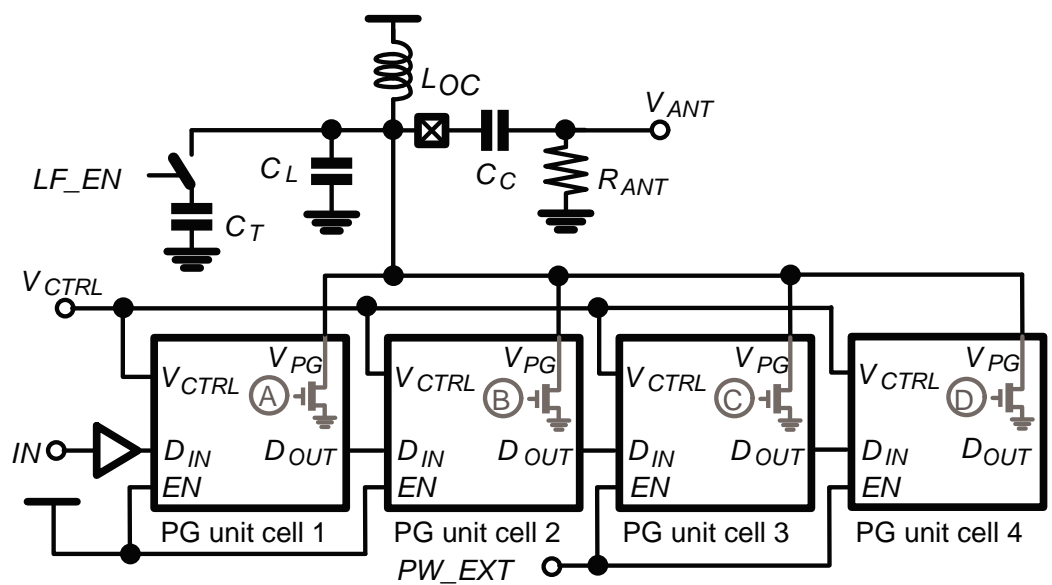

(a)

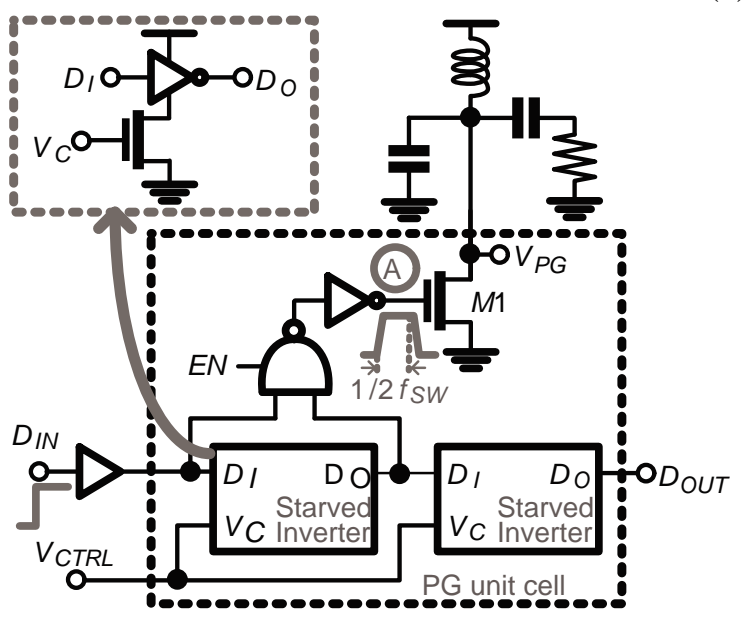

(b)

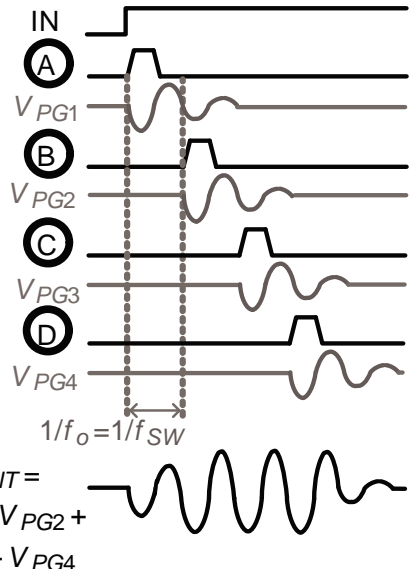

(c)

Figure 4. (a) The proposed PG, (b) the PG unit cell, and (c) the PG operation. 
in sequence with a frequency of approximately $f_{S W}$ by using a DL. Energy is then injected to an RLC tank consisting of $R_{A N T}$, an on-chip inductor $L_{O C}$, a tuning capacitor $\left(C_{T}\right)$ and loading and parasitic capacitors $\left(C_{L}\right)$, with a small $Q_{T}$ of two because of the insertion loss and mainlobe $\mathrm{BW}$ considerations. The DL approach is adopted because DLs are usually simpler and more energy-efficient than oscillators.

The PG is constructed by cascading four unit cells. The schematic of unit cell is shown in Figure 4(b). The PD (with a length of approximately $\frac{1}{2 f_{S W}}$ ) of a starved inverter is used to generate an impulse with a width of approximately $\frac{1}{2 f_{S W}}$ at node A and M1 is turned on. This pulls down the output voltage and injects energy into the tank. Due to the $R_{A N T}$, the signal decays as indicated from (3). If $f_{S W}$ is approximately equal to $f_{o}$ by setting the control voltage of the starved inverter $\left(V_{C}\right)$, the output signals from all unit cells are in phase and added together. As a result, a higher-energy and longer-PW UWB pulse can be generated. The idea is depicted in Figure 4(c). Note that the waveforms $V_{P G i}\left(V_{P G 1}-V_{P G 4}\right.$, which are highlighted in grey color) represent the output signals contributed by the $i$-th unit cell, they are shown for illustration purposes and do not exist actually in the system.

One drawback of other DL-based MPC PGs $[7,8]$ is that $f_{C}$ and $\mathrm{BW}$ are sensitive to the PD variation of the DL. The proposed PG is relatively less sensitive to this variation because the tank bandpass property concentrates the output energy at $f_{o}$. Figure 5 shows the system-level simulated power spectra with $\pm 5 \%$ mismatches between $f_{S W}$ and $f_{o}$. Around $\pm 3 \%$ variation on $f_{C}$ and $\pm 4 \%$ variation on $B W_{-10 \mathrm{~dB}}$ are observed. Larger $Q_{T}$ can make the PG less sensitive to the PD variation, with trade-offs as aforementioned.

The output parasitic capacitance which is usually dominating inside the system is tuned out by $L_{O C}$, the driving requirement and, hence, the transistors size of the output driver are reduced. In other words, the capacitive and switching losses are also reduced which makes the designed PG energyefficient. $L_{O C}$ connects the PG output to the positive supply rail at DC which provides electrostatic discharge protection.

Another advantage of the proposed PG is its high flexibility. The PW is scalable by adding more unit cells. If more unit cells are cascaded in series, smaller BW and higher-energy outputs can be generated. In addition, the output energy, $f_{C}$ and $B W_{-10 \mathrm{~dB}}$ (hence also PW) can be adjusted depending on channel conditions. If the radios nearby are using similar operating frequency, $f_{C}$ can be reduced by decreasing $V_{C T R L}$ and setting LF_EN to ' 1 ' to connect $C_{T}$. More frequency steps can be obtained by replacing $C_{T}$ with a switched-capacitor array. If the interference from other radios becomes less significant, some unit cells can be disabled by setting their EN input to ' 0 '. The energy consumption can then be reduced. In the designed PG, either two or four unit cells are enabled by switching PW_EXT.

\section{EXPERIMENTAL RESULTS}

A proof-of-concept prototype is implemented in a TSMC $90 \mathrm{~nm}$ CMOS process. A chip microphotograph is shown in Figure 6. The core area excluding pads is $0.092 \mathrm{~mm}^{2}$. The supply voltage is $1.2 \mathrm{~V}$. The PG

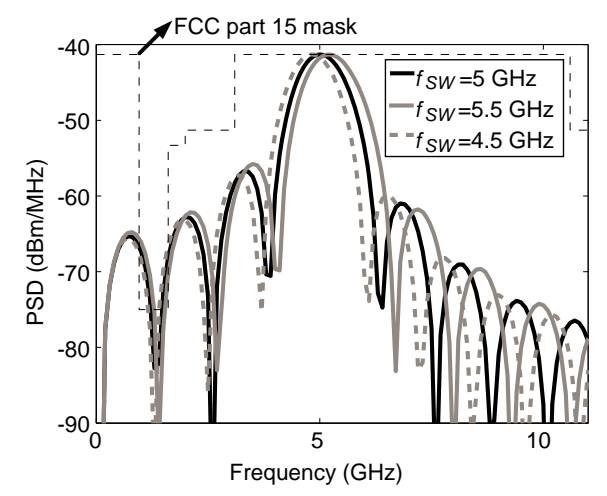

Figure 5. Power spectra with different mismatches between $f_{o}$ and $f_{S W}\left(f_{o}=5 \mathrm{GHz}\right)$.

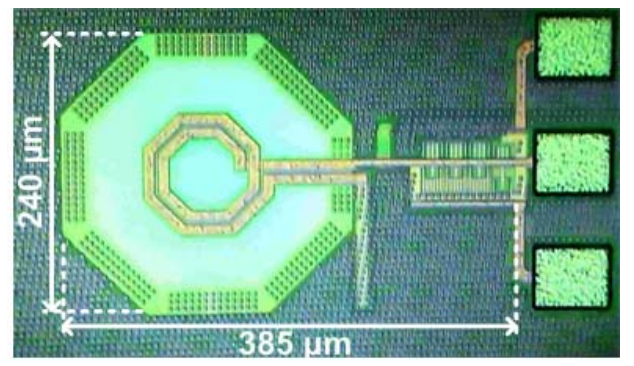

Figure 6. A Chip microphotograph. 
output is probed on-chip and connected to a $50 \Omega$ load. Four different settings are tested:

(i) LF_EN $=$ ' 0 ' and PW_EXT $=$ ' 1 ': All unit cells are enabled $(N=4)$ with high $f_{C}$.

(ii) LF_EN $=$ ' 1 ' and PW_EXT $=$ ' 1 ': All unit cells are enabled $(N=4)$ with low $f_{C}$.

(iii) LF_EN = ' 0 ' and PW_EXT = ' 0 ': Two unit cells are enabled $(N=2)$ with high $f_{C}$.

(iv) LF_EN $=$ ' 1 ' and PW_EXT $=$ '0': Two unit cells are enabled $(N=2)$ with low $f_{C}$.

$V_{C T R L}$ is changed accordingly when LF_EN $=$ ' 1 '. The resultant timing waveforms and power spectra with $80 \mathrm{MHz}$ pulse repetition rate $(\mathrm{PRF})$ are shown in Figure 7 and Figure 8 respectively. $f_{C}$ can be adjusted from $4 \mathrm{GHz}$ to $5.6 \mathrm{GHz}$, a larger peak energy and a narrower $\mathrm{BW}$ can be achieved by adding more unit cells (i.e., larger $N)$. The LF components $(<900 \mathrm{MHz})$ are due to the ringing caused by the bondwires of the voltage supplies, these components can be removed by adding decoupling capacitors. The proposed PG achieves a LF sidelobe $(<2 \mathrm{GHz})$ energy reduction of $15-20 \mathrm{~dB}$. Some LF sidelobes violate the FCC part 15 mask at such high PRF, we assume they will be removed by adding HPF or simply exploring the antenna bandpass property.

The PG performance is summarized and compared with other published state-of-the-art IR-UWB PGs in Table 1. The PG energy efficiency is commonly used to compare different PG performance $[9,11]$ and given as:

$$
\eta=\frac{\frac{V_{P-P}^{2}}{8 R_{L}} P W}{E_{C}}
$$

where $V_{P-P}$ is the peak-to-peak voltage, $R_{L}$ is the load resistance and $E_{C}$ is the energy consumption per pulse. Nevertheless it is difficult to define the PW precisely because of the ringing caused by the
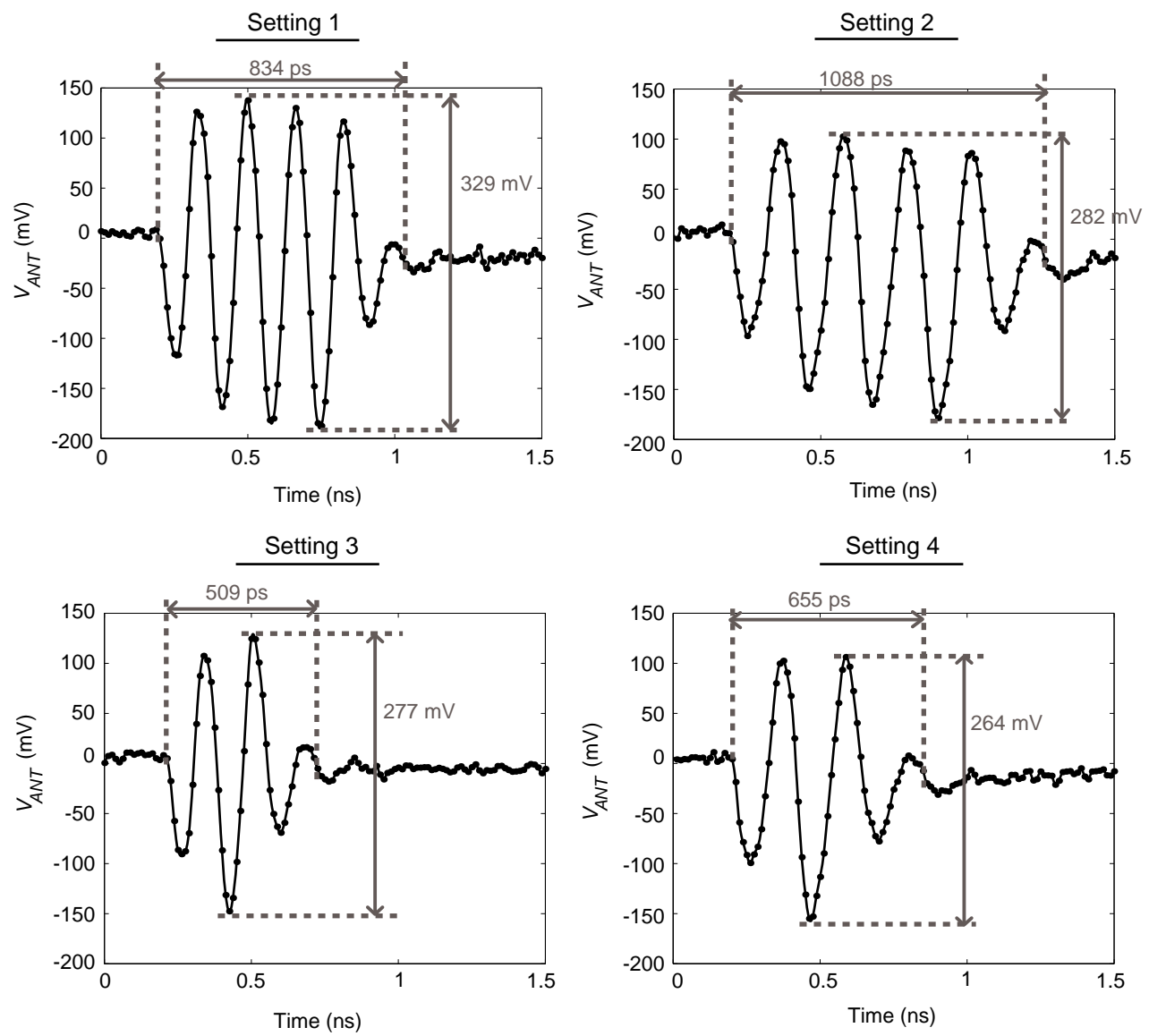

Figure 7. Timing waveforms of the PG output. 


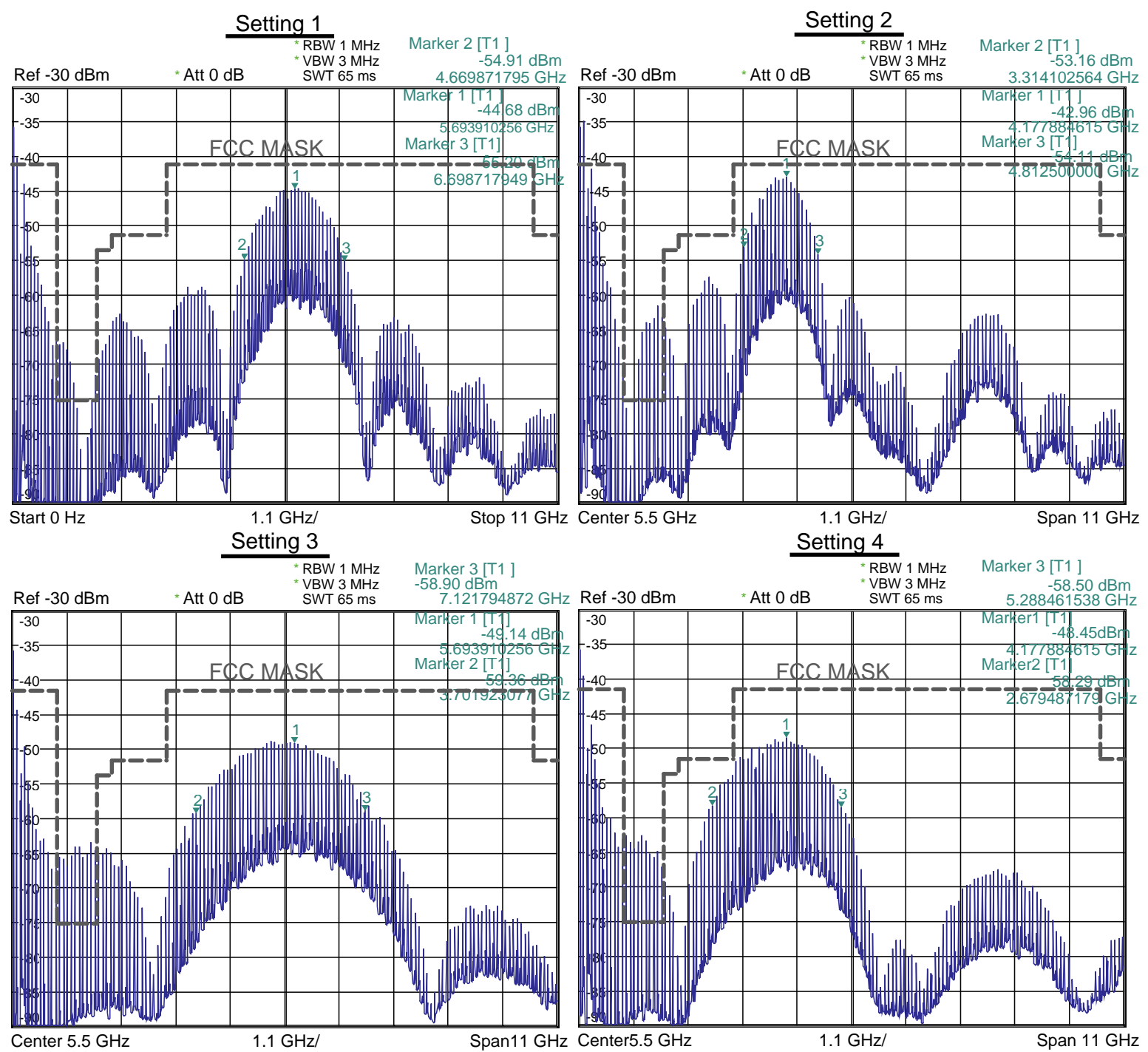

Figure 8. Power spectra of the PG output.

Table 1. A comparison with other published IR-UWB PGs.

\begin{tabular}{|c|c|c|c|c|c|c|c|c|c|c|c|}
\hline & \multirow{2}{*}{ [9] } & \multirow{2}{*}{ [11] } & \multirow{2}{*}{ [7] } & \multirow{2}{*}{ [4] } & \multirow{2}{*}[3]{} & \multirow{2}{*}{ [8] } & \multirow{2}{*}{ [5] } & \multicolumn{4}{|c|}{ This work } \\
\hline & & & & & & & & $\begin{array}{l}\text { Setting } 1 \\
\end{array}$ & 2 & 3 & 4 \\
\hline Technology & $\begin{array}{l}180 \mathrm{~nm} \\
\text { CMOS }\end{array}$ & $\begin{array}{l}2 \mu \mathrm{m} \\
\mathrm{HBT}\end{array}$ & $\begin{array}{c}90 \mathrm{~nm} \\
\text { CMOS }\end{array}$ & $\begin{array}{l}180 \mathrm{~nm} \\
\text { CMOS }\end{array}$ & $\begin{array}{l}180 \mathrm{~nm} \\
\text { CMOS }\end{array}$ & $\begin{array}{l}180 \mathrm{~nm} \\
\text { CMOS }\end{array}$ & $\begin{array}{l}130 \mathrm{~nm} \\
\text { CMOS }\end{array}$ & \multicolumn{4}{|c|}{$\begin{array}{c}90 \mathrm{~nm} \\
\mathrm{CMOS}\end{array}$} \\
\hline Modulation & OOK & OOK & OOK & OOK & OOK & OOK & BPSK & \multicolumn{4}{|c|}{ OOK } \\
\hline$E_{C}(\mathrm{pJ} / \mathrm{pulse})$ & 118 & 790 & 5.2 & 28 & 9.2 & 20 & 51.5 & 5.2 & 7.5 & 2.8 & 4 \\
\hline$V_{P-P}(\mathrm{~V})$ & $4.9^{\mathrm{a}}$ & 6.2 & 0.41 & 0.67 & 0.22 & 0.26 & 0.19 & 0.33 & 0.28 & 0.28 & 0.26 \\
\hline PW (ns) & 1 & $\approx 1^{\mathrm{b}}$ & 0.96 & 0.5 & 0.7 & 1 & 0.5 & 0.83 & 1.1 & 0.51 & 0.66 \\
\hline$B W_{-10-\mathrm{dB}}(\mathrm{GHz})$ & 2 & 1.8 & 1.9 & 4 & $\approx 3.2^{\mathrm{b}}$ & 2 & $\approx 3.8^{\mathrm{b}}$ & 2 & 1.5 & 3.4 & 2.6 \\
\hline$f_{C}(\mathrm{GHz})$ & 4.3 & 4.1 & 4 & 8 & $\approx 3.9^{\mathrm{b}}$ & 4 & $\approx 8.3^{\mathrm{b}}$ & 5.6 & 4.1 & 5.4 & 4 \\
\hline$\eta(\%)$ & 25.4 & 10.4 & 7.9 & 2 & 1 & 0.1 & 0.01 & 4.3 & 2.9 & 3.5 & 2.9 \\
\hline FoM $(\%)$ & 12.7 & 6.8 & 4.3 & 1 & 0.4 & 0.4 & 0.05 & 2.6 & 1.7 & 2.1 & 1.6 \\
\hline Area $\left(\mathrm{mm}^{2}\right)$ & 0.19 & 1 & 0.0015 & 0.11 & N/A & 0.08 & N/A & \multicolumn{4}{|c|}{0.09} \\
\hline
\end{tabular}


bondwires. Also, (8) assumes the PG output is a sinewave burst and ignores the pulse shape. To improve this, a new figure-of-merit (FoM) is proposed and given as

$$
\mathrm{FoM}=\frac{\frac{V_{P-P}^{2}}{8 R_{L}} \cdot \frac{1}{B W_{-10 \mathrm{~dB}}}}{E_{C}}
$$

Modern spectrum analyzers can measure signal BWs precisely, and $B W_{-10 \mathrm{~dB}}$ can partly represents the pulse shape, hence a more precise comparison can be made.

The proposed PG is one of the few candidates that can achieve $E_{C}$ of smaller than $10 \mathrm{pJ} / \mathrm{pulse}$. Competitive FoM and $\eta$ are measured, FoM ranges from $1.6 \%$ to $2.6 \%$ and $\eta$ ranges from $2.9 \%$ to $4.3 \%$. In [7], a DL-based MPC PG with $E_{C}=5.2 \mathrm{pJ} /$ pulse and FoM $=4.3 \%$ has been reported. Nevertheless it is relatively more sensitive to the PD variation of the DL as aforementioned. A BPSK PG using spectrum mixing approach has been reported in [5]. The BPSK modulations may improve the system performance, however, a very low FoM of $0.05 \%$ has been reported because the PG structure is similar to conventional narrowband TXs.

In [9], a SO topology with a very high FoM of $12.7 \%$ has been demonstrated. However, the measured $E_{C}$ is $118 \mathrm{pJ} /$ pulse, we assume the large $E_{C}$ is due to the fact that large current (peak current of $70 \mathrm{~mA}$ ) is needed to start-up the low-QF LC oscillator and maximize the output swing (and hence $\eta$ ). In [11], an impulse-forming approach has been proposed and a FoM of $6.8 \%$ has been reported. The idea is similar to distributing amplifier, an impulse is inputted to an off-chip tapped DL network which consists of transmission lines and bondwire inductors. The impulse and its replicas are then delayed, scaled and combined at the PG output. The large FoM is because of the usage of HBT transistors which provides higher power capacity and high speed. However, this PG is not suitable for wireless-powered IR-UWB radios because of its large $E_{C}$ of $790 \mathrm{pJ} /$ pulse and large area due to the pad and bondwire inductors. Also, the HBT process fabrication is more expensive compared to the CMOS counterparts.

\section{CONCLUSION}

A low-power energy-efficient OOK IR-UWB PG intended for wireless-powered IR-UWB radio applications has been presented. The output power, $f_{C}$ and $\mathrm{PW}$ are controllable depending on channel conditions and data rates. Qualitative frequency-domain and transient analyses have been presented and matched with the measurement results. A new FoM has been proposed to compare different PGs in a more precise way. A proof-of-concept prototype has been successfully implemented in a TSMC $90 \mathrm{~nm}$ CMOS process. The core area is $0.092 \mathrm{~mm}^{2}$. Measurements show the energy consumption and FoM to be $2.8-7.5 \mathrm{pJ} /$ pulse and 1.6-2.6\%, respectively, which is competitive to most published IR-UWB PGs and proves the proposed PG suitable for wireless-powered radios. The output swing is $277-329 \mathrm{mV} \mathrm{V}_{\mathrm{p}-\mathrm{p}}$. $\mathrm{PW}$ and $f_{C}$ range 509-1088 ps and $4-5.6 \mathrm{GHz}$, respectively, which provides freedoms to optimize the system performance.

\section{ACKNOWLEDGMENT}

The authors would like to thank Kristian G. Kjelgård, Olav S. Kyrvestad, Prof. Philipp D. Häfliger and Novelda AS for technical discussion and support. This work was supported in part by the Norwegian Research Council through the Norwegian Ph.D. Network on Nanotechnology for Microsystems (contract No. 190086/S10).

\section{REFERENCES}

1. Lee, K. K. and T. S. Lande, "A wireless-powered IR-UWB transmitter for long-range passive RFID tags in 90-nm CMOS," IEEE Transactions on Circuits and Systems II: Express Briefs, Vol. 61, No. 11, 870-874, Nov. 2014.

2. Radiom, S., et al., "Far-field on-chip antennas monolithically integrated in a wireless-powered $5.8 \mathrm{GHz}$ downlink/UWB uplink RFID tag in 0.18- $\mu \mathrm{m}$ standard CMOS," IEEE Journal of SolidState Circuits, Vol. 45, No. 9, 1746-1758, Sep. 2010. 
3. Baghaei-Nejad, M., et al., "A remote-powered RFID tag with $10 \mathrm{Mb} / \mathrm{s}$ UWB uplink and $-18.5 \mathrm{dBm}$ sensitivity UHF downlink in $0.18 \mu \mathrm{m}$ CMOS," IEEE ISSCC Digest of Technical Papers, 198-199, Feb. 2009.

4. Sim, S., D.-W. Kim, and S. Hong, "A CMOS UWB pulse generator for 6-10 GHz applications," IEEE Microwave and Wireless Components Letters, Vol. 19, No. 2, 83-85, Feb. 2009.

5. Zhou, L., et al., "A 2-Gb/s 130-nm CMOS RF-correlation-based IR-UWB transceiver front-end," IEEE Transactions on Microwave Theory and Techniques, Vol. 59, No. 4, 1117-1130, Jan. 2011.

6. Ryckaert, J., et al., "A 0.65-to-1.4 nJ/burst 3-to-10 GHz UWB all-digital TX in $90 \mathrm{~nm}$ CMOS for IEEE 802.15.4a," IEEE Journal of Solid-State Circuits, Vol. 42, No. 12, 2860-2869, Dec. 2007.

7. Lee, K. K., M. Z. Dooghabadi, H. A. Hjortland, Ø. Næss, and T. S. Lande, "A 5.2 pJ/pulse impulse radio pulse generator in $90 \mathrm{~nm}$ CMOS," Proceedings of IEEE International Symposium on Circuits and Systems, 1299-1302, May 2011.

8. Zhao, M. J., B. Li, and Z. H. Wu, "20-pJ/pulse 250 Mbps low-complexity CMOS UWB transmitter for 3-5 GHz applications," IEEE Microwave and Wireless Components Letters, Vol. 23, No. 3, 158-160, Mar. 2013.

9. Diao, S. X., Y. J. Zheng, and C. H. Heng, "A CMOS ultra low-power and highly efficient UWBIR transmitter for WPAN applications," IEEE Transactions on Circuits and Systems II: Express Briefs, Vol. 56, No. 3, 200-204, Mar. 2009.

10. Niknejad, A. M., Electromagnetics for High-speed Analog and Digital Communication Circuits, Cambridge University Press, Cambridge, 2007.

11. Xia, J., C. L. Law, Y. Zhou, and K. S. Koh, " $3-5 \mathrm{GHz}$ UWB impulse radio transmitter and receiver MMIC optimized for long range precision wireless sensor networks," IEEE Transactions on Microwave Theory and Techniques, Vol. 58, No. 12, 4040-4051, Dec. 2010. 